\title{
Nonlinear vectorial two-beam coupling and forward four-wave mixing in photorefractive materials
}

\author{
Baruch Fischer \\ Department of Electrical Engineering, Technion-Israel Institute of Technology, Haifa 32000, Israel \\ Jeffrey $O$. White \\ Hughes Research Laboratories, Malibu, California 90265 \\ Mark Cronin-Golomb \\ Ortel Corporation, 2015 West Chestnut Street, Alhambra, California 91803 \\ Amnon Yariv \\ California Institute of Technology, Pasadena, California 91125
}

Received October 25, 1985; accepted January 28, 1986

\begin{abstract}
We present an exact solution of a nonlinear vector analysis of two-beam coupling and forward four-wave mixing in photorefractive media.
\end{abstract}

In this Letter we present an exact solution with linear absorption for a system of nonlinear differential equations that model two separate photorefractive processes. The first is the coupling of two beams with general polarizations, restricted to the case when the magnitude of the coupling for one polarization is the same as that for the other polarization. ${ }^{1}$ The second is scalar forward four-wave mixing, ${ }^{2}$ which has practical importance when noncounterpropagating phase conjugation is required, as in the case of image transmission through two lengths of equivalent optical fiber. ${ }^{3}$

When a pair of beams writes a grating in a photorefractive medium, that grating in its turn affects the propagation of the writing beams, which are thus coupled nonlinearly to each other., 4 The nonlinear coupled-wave equations that describe the interaction in the scalar case-when the two beams have the same polarizations-have been solved analytically. 4,5 This solution describes the energy exchange between the beams when a phase shift exists between the light interference fringe pattern and the refractive-index grating and is the basic cornerstone to an understanding of the broader effects of four-wave mixing in photorefractive materials. ${ }^{6}$

The situation becomes more complicated when one considers the interaction of two beams with general polarizations. Four coupled nonlinear differential equations then arise, describing the interactions among the four components of the beams' optical electric fields. The nonlinear source term from the selfinduced grating causes the overall coupling of the equations. Our solution for these equations is valid in the case when direct cross coupling between vertical and horizontal polarization is negligible. This will often be the case in practical situations, where bire- fringence does not allow phase matching for such couplings. We also require that the magnitude of the coupling constants be the same for both polarizations. Mapping this problem to the known solution of fourwave mixing in photorefractive materials ${ }^{6,7}$ will let us follow similar mathematical procedures toward an explicit analytical solution including the effects of linear absorption.

The vectorial interaction geometry that we consider is shown in Fig. 1. Two beams with frequency $\omega$ propagate in the crystal from $z=0$ to $z=l$. The vectorial field amplitude of each beam is represented by two orthogonal components. $A_{1}$ and $A_{2}$ are the components of the first beam, and $A_{4}$ and $A_{3}$ are components of the second one. Note that the first components of

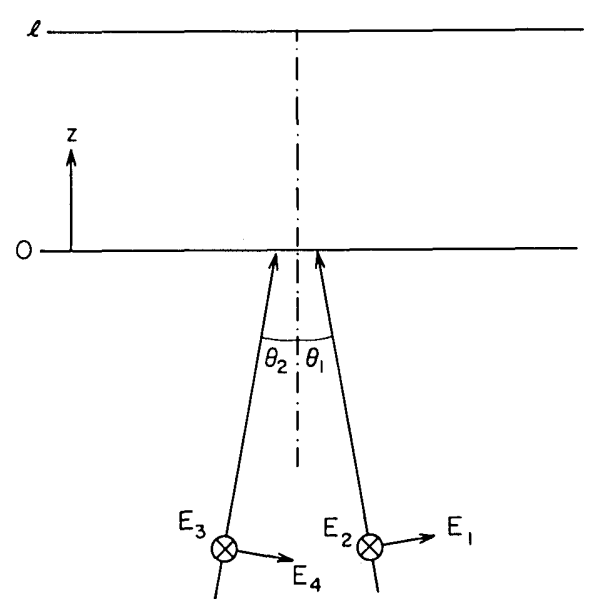

Fig. 1. Vectorial two-wave mixing geometry analyzed in text. 
the two beams are not parallel. As in Ref. 3 we obtain the following coupled-wave equations, neglecting, for the moment, linear absorption:

$$
\begin{aligned}
\frac{\mathrm{d} A_{1}}{\mathrm{~d} z} & =-b_{\mathrm{I}} g A_{4}-b_{\mathrm{II}} g A_{3}, \\
\frac{\mathrm{d} A_{2}}{\mathrm{~d} z} & =-b_{\mathrm{I}} g A_{3}-b_{\mathrm{II}} g A_{4}, \\
\frac{\mathrm{d} A_{3}^{*}}{\mathrm{~d} z} & =b_{\mathrm{I}} g A_{2}{ }^{*}+b_{\mathrm{II}} g A_{1}^{*}, \\
\frac{\mathrm{d} A_{4}^{*}}{\mathrm{~d} z} & =b_{\mathrm{I}} g A_{1}{ }^{*}+b_{\mathrm{II}} g A_{2}{ }^{*},
\end{aligned}
$$

where

$$
g=\frac{A_{1} A_{4}{ }^{*}+A_{2} A_{3}{ }^{*}}{I_{0}},
$$

$I_{0}$ is the total spatially averaged light intensity

$$
I_{0}=A_{1} A_{1}{ }^{*}+A_{2} A_{2}{ }^{*}+A_{3} A_{3}{ }^{*}+A_{4} A_{4}{ }^{*} \text {, }
$$

and $b_{\mathrm{I}}$ and $b_{\mathrm{II}}$ are the parallel and cross-component couplings between the two beams. These constants generally depend on material parameters such as the temperature, grating period and orientation, and dielectric and electro-optic tensors of the material.5,6 They are approximately independent of the intensities of the interacting beams. Equations (1) may be used to show the conservation of total intensity, so that $I_{0}$ is constant. Henceforth, we normalize the equations so that $I_{0}=1$.

Equivalence of these equations to those of fourwave mixing in photorefractive materials ${ }^{6,7}$ arises if we retain only the first terms on the right-hand sides of Eqs. (1) so that we assume that only parallel coupling is important. Then the substitutions $A_{2} \rightarrow A_{2}{ }^{*}$ and $A_{3} \rightarrow A_{3}{ }^{*}$ transform one system into the other. In that case $\left(b_{\mathrm{II}}=0\right)$, we are able to solve the equations exactly in the same way as in Ref. 7.

With the conservation laws $\left(I_{j}=\left|A_{j}\right|^{2}\right)$,

$$
\begin{aligned}
I_{1}+I_{4} & =d_{1}, \\
I_{2}+I_{3} & =d_{2}, \\
A_{1} A_{2}{ }^{*}+A_{3}{ }^{*} A_{4} & =c,
\end{aligned}
$$

we obtain

$$
\begin{gathered}
\frac{\mathrm{d}}{\mathrm{d} z}\left(\frac{A_{1}}{A_{2}}\right)=-b_{\mathrm{I}}\left[c+\left(d_{1}-d_{2}\right)\left(\frac{A_{1}}{A_{2}}\right)-c^{*}\left(\frac{A_{1}}{A_{2}}\right)^{2}\right], \\
\frac{\mathrm{d}}{\mathrm{d} z}\left(\frac{A_{3}}{A_{4}}\right)=b_{\mathrm{I}}\left[c+\left(d_{2}-d_{1}\right)\left(\frac{A_{3}}{A_{4}}\right)-c^{*}\left(\frac{A_{3}}{A_{4}}\right)^{2}\right] .
\end{gathered}
$$

These equations may be immediately integrated to give

$$
\begin{aligned}
& \frac{A_{1}}{A_{2}}=-\left[\frac{D S_{-} e^{-\mu z}-D^{-1} S_{+} e^{\mu z}}{2 c^{*}\left(D e^{-\mu z}-D^{-1} e^{\mu z}\right)}\right], \\
& \frac{A_{3}}{A_{4}}=\left[\frac{E S \_e^{-\mu z}-E^{-1} S_{+} e^{\mu z}}{2 c^{*}\left(E e^{-\mu z}-E^{-1} e^{\mu z}\right)}\right],
\end{aligned}
$$

where

$$
\begin{gathered}
S_{ \pm}=\Delta \pm\left(\Delta^{2}+4|c|^{2}\right)^{1 / 2}, \\
\mu=\frac{b_{\mathrm{I}}}{2}\left(\Delta^{2}+4|c|^{2}\right)^{1 / 2},
\end{gathered}
$$

and

$$
\Delta=d_{2}-d_{1} .
$$

In contrast to the four-wave mixing case, all the boundary conditions are set at the same boundary because all the beams are propagating in the same (positive- $z$ ) direction. The constants of integration $\Delta$, $c, D$, and $E$ can thus be readily obtained, and, for the same reason, we can include linear absorption in our system without changing the equations if we assume that the absorption coefficients for each polarization are the same. The linear absorption terms $-\alpha A_{j}$ that are added in Eqs. (1) can be eliminated mathematically by the transformation $A_{j} \rightarrow A_{j} e^{\alpha z}$, remembering that in this transformation we also have $I_{0} \rightarrow I_{0} e^{2 \alpha z}$.

Given all $A_{j}(z=0)=A_{j}(0)$, we get

$$
\begin{aligned}
& D^{2}=\frac{2 c^{*} A_{12}(0)+S_{+}}{2 c^{*} A_{12}(0)+S_{-}}, \\
& E^{2}=\frac{2 c^{*} A_{34}(0)-S_{+}}{2 c^{*} A_{34}(0)-S_{-}},
\end{aligned}
$$

where $A_{i j}=A_{i} / A_{j}$. Together with the constants

$$
\begin{aligned}
\Delta & =I_{2}(0)+I_{3}(0)-I_{1}(0)-I_{4}(0), \\
c & =A_{1}(0) A_{2}{ }^{*}(0)+A_{3}{ }^{*}(0) A_{4}(0)
\end{aligned}
$$

we can obtain the ratios $A_{12}(z)$ and $A_{34}(z)$. Then the beam intensities can be expressed by these ratios and the conservation laws

$$
I_{1}(z)=I_{12}(z)\left[\frac{d_{2}-I_{34}(z) d_{1}}{1-I_{12}(z) I_{34}(z)}\right],
$$

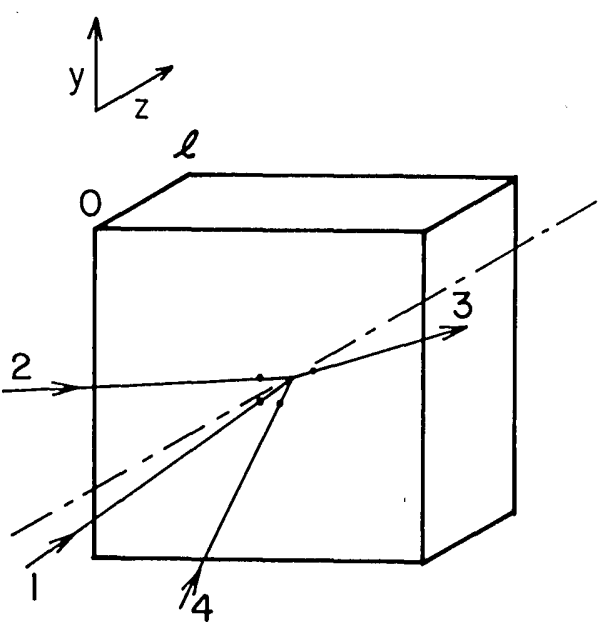

Fig. 2. Forward four-wave mixing geometry analyzed in text. 


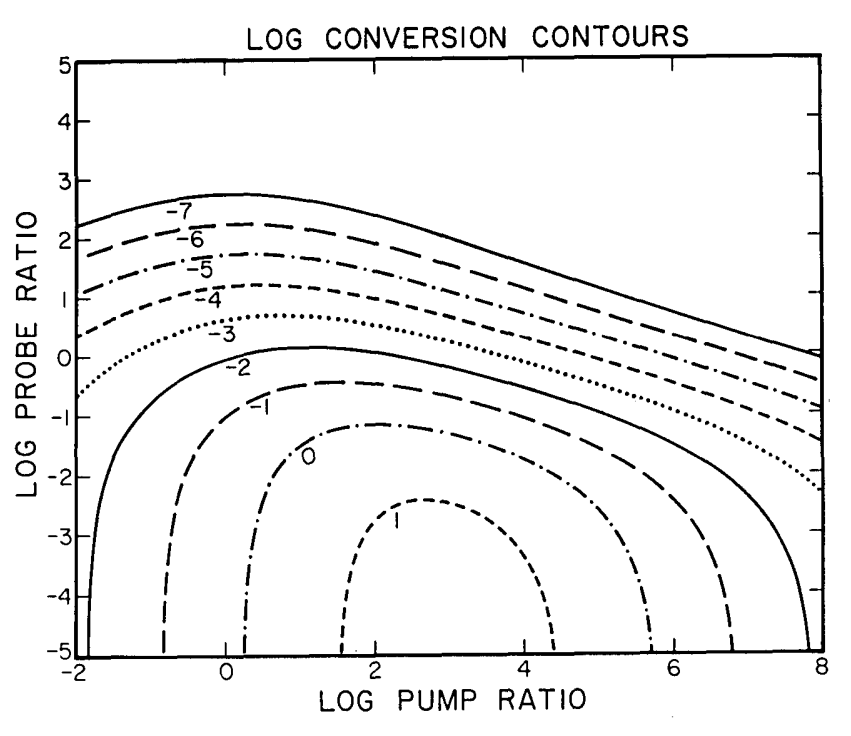

Fig. 3. Contour plot of $\log$ forward phase-conjugate conversion efficiency for coupling constant $b_{\mathrm{I}} l=3$ versus pump and probe ratio logarithms.

$$
\begin{aligned}
& I_{2}(z)=\left[\frac{d_{2}-I_{34}(z) d_{1}}{1-I_{12}(z) I_{34}(z)}\right], \\
& I_{3}(z)=I_{34}(z)\left[\frac{d_{1}-I_{12}(z) d_{2}}{1-I_{12}(z) I_{34}(z)}\right], \\
& I_{4}(z)=\left[\frac{d_{1}-I_{12}(z) d_{2}}{1-I_{12}(z) I_{34}(z)}\right] .
\end{aligned}
$$

These results also apply to the case of forward fourwave mixing phase conjugation in the scalar case, as shown in Fig. 2. $A_{1}$ is distinguished from $A_{2}$ and $A_{4}$ is distinguished from $A_{3}$ by giving the wave vectors of the pair of beams 1 and 3 a vertical $y$ component slightly different from that of the pair of beams 4 and 2 . Again we have to assume that cross coupling is negligible. Figure 3 shows a contour plot of phase-conjugate conversion efficiency as a function of the pump ratio $r$ $=I_{2}(0) / I_{1}(0)$ and the probe ratio $q=I_{4}(0) /\left[I_{1}(0)+\right.$ $\left.I_{2}(0)\right]$. We note that for small $q$ the solution reduces to that of the regular undepleted-pumps approximation for photorefractive four-wave mixing. ${ }^{6}$ Also, the result appears similar to that for regular four-wave mixing by the reflection grating, ${ }^{8}$ with the exception that the forward four-wave mixing case has lower conversion efficiencies.

In summary, we have presented nonlinear solutions for photorefractive vectorial two-beam coupling and for forward phase conjugation in photorefractive crystals. The result for forward phase conjugation may have practical importance for image transmission through optical fibers.

This research was supported by the U.S. Air Force Office of Scientific Research and the U.S. Army Research Office, Durham, North Carolina.

\section{References}

1. We note that in many cases of practical importance in $\mathrm{BaTiO}_{3}$ and $\mathrm{LiNb}_{2} \mathrm{O}_{6}$, for example, the couplings for the different polarizations are not the same.

2. A. Khyzniak, V. Kondilenko, Yu. Kucherov, S. Lesnik, S. Odulov, and M. Soskin, J. Opt. Soc. Am. A 1, 169 (1984).

3. A. Yariv, Appl. Phys. Lett. 28, 88 (1976).

4. D. W. Vahey, J. Appl. Phys. 46, 3510 (1975).

5. N. V. Khuktarev, V. B. Markov, S. G. Odulov, M. S. Soskin, and L. Vinetskii, Ferroelectrics 22, 949 (1979).

6. B. Fischer, M. Cronin-Golomb, J. O. White, and A. Yariv, Opt. Lett. 6, 519 (1981).

7. M. Cronin-Golomb, B. Fischer, J. O. White, and A. Yariv, Opt. Lett. 7, 313 (1982).

8. M. Cronin-Golomb, B. Fischer, J. O. White, and A. Yariv, IEEE J. Quantum Electron. QE-20, 12 (1984). 